\title{
Viscoelasticity Measurements by an Optofluidic Micro-Rheometer
}

\author{
V. Vitali ${ }^{1}$, G. Nava ${ }^{2}$, G. Zanchetta ${ }^{2}$, F. Bragheri ${ }^{3}$, R. Osellame ${ }^{3}$, T. Bellini ${ }^{2}$ I. Cristiani $^{1}$, P. Minzioni ${ }^{1}$ \\ 1. Department of Electrical, Computer, and Biomedical Engineering, Università di Pavia, Via Ferrata 5A, 27100, Pavia, Italy \\ 2. Department of Medical Biotechnology and Translational Medicine, Università di Milano, Via F.lli Cervi 91, 20090 Segrate, Italy \\ 3. Istituto di Fotonica e Nanotecnologie (IFN), CNR, Piazza Leonardo da Vinci 32, 20133 Milano, Italy
}

During the last decades, microrheology attracted a significant attention thanks to the possibility of investigating the viscoelastic properties of complex systems (e.g. cells and soft materials) at micrometer scale. The inherent low-consumption of sample offered by microrheology makes it the ideal candidate to study the rheological properties of precious/limited materials. In active microrheology, optical or magnetic forces enable trapping and manipulation of micro-probes in the fluid under test. The probe's response to external stimuli is used to derive the rheological properties of the surrounding medium. While this approach has been already reported in the scientific literature mainly using optical tweezers [1], in this document we propose a different system configuration based on a dual beam laser trap, previously exploited to realize a simple viscometer $[2,3]$. The here proposed device has all the features of a rheometer, also allowing to measure the elastic properties, and has the advantage of requiring a lower beam intensity while being able to apply larger forces with respect to standard optical tweezers. Additionally the system can be easily integrated in a glass substrate, requiring just an external connection to a CW-laser source and a low-magnification objective for sample observation.

The measurement idea consists in the sinusoidal modulation of the optical power emitted by two facing waveguides, with an opposite phase, thus causing a controlled oscillation of a trapped microbead. By simultaneous measurements of both the particle position and the optical force, the phase difference $(\Delta \varphi)$ between the force and position signals is retrieved, yielding information about the system viscosity and elasticity, see Fig.1 (left). Additionally, measurements can be easily carried out at different oscillation frequencies (up to $10 \mathrm{~Hz}$ with current system) and at different temperatures. The system has been initially calibrated with standard Newtonian fluids. The micro-rheometer performances were demonstrated by measuring an aqueous wormlike micellar solution $(100 \mathrm{mM}$ cetylpyridinium chloride/ $50 \mathrm{mM}$ sodium salicylate which has well-known viscoelastic properties) obtaining the results reported in Fig. 1. Top and bottom panels on the left side of Fig.1 show the evident dependence of $\Delta \varphi$ on oscillation frequency: the decrease of $\Delta \varphi$ shows that the system is passing from a viscosity-dominated regime to an elasticity-dominated regime. On the right panel of Fig. 1 the derived viscosity $\eta$ and the elastic modulus $\mathrm{E}$ as a function of frequency are reported.
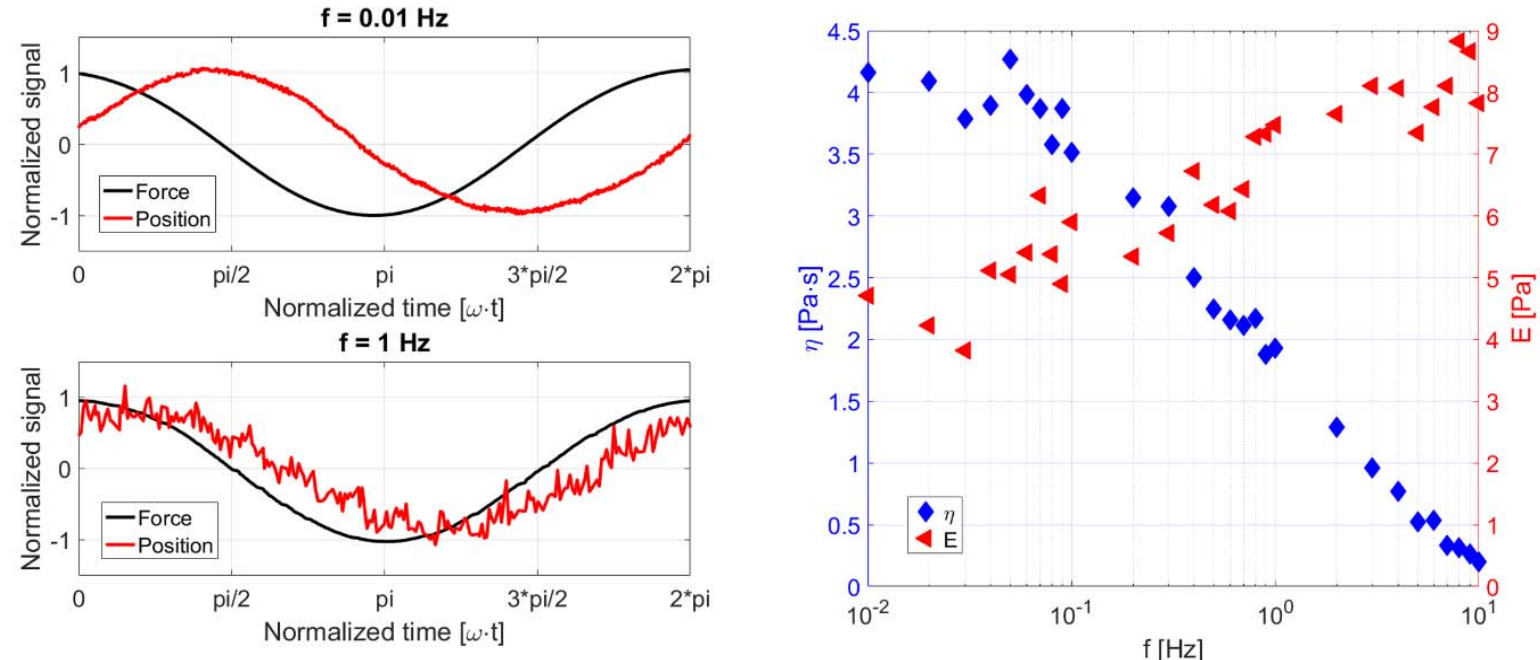

Fig. 1 Results from the measurements on the aqueous wormlike micellar solution. Left: normalized force and position signals measured at two different oscillation frequencies; Right: viscosity $\eta$ and elastic modulus $E$ as a function of the oscillation frequency.

\section{References}

[1] A. Yao, M. Tassieri, M. Padgett, J. Cooper, "Microrheology with optical tweezers," Lab Chip 9, 2568-2575 (2009).

[2] T. Yang, G. Nava, V. Vitali, F. Bragheri, R. Osellame, T. Bellini, I. Cristiani, P. Minzioni, "Integrated Optofluidic Chip for Low-Volume Fluid Viscosity Measurement," Micromachines 8, 65 (2017).

[3] G. Nava, T. Yang, V. Vitali, P. Minzioni, I. Cristiani, F. Bragheri, R. Osellame, L. Bethge, S. Klussmann, E. M. Paraboschi, R. Asselta, T. Bellini, "Newtonian to non-newtonian fluid transition of a model transient network," Soft Matter 14, 3288-3295 (2018). 